Research Article

\title{
Improving Comparative Sentence Extraction of Chinese Product Reviews by Sentiment Analysis
}

\author{
Lulin Shi, Shi Li *, Ping Jiang and Hongsen Liu
}

School of Information and Computer Engineering, Northeast Forestry University, Harbin 150040, PR China

Received 19 August 2016; Accepted 27 November 2016

\begin{abstract}
Comparative sentences in online reviews express substantial information that are of concern to customers and attract increasing attention from manufacturers and service providers. At present studies of Chinese Comparative Sentence Identification are based on Pattern Matching and Supervised Machine Learning Algorithm, in which its performance requires further improvement. Therefore, the present analysis aims to further identify the candidate sets of comparative sentences by unsupervised sentiment analysis, and then improve mining performance. First, we constructed a Chinese comparative pattern set, which was used to extract candidate comparative sentences from the corpus of online products reviews. Moreover, we set the score for the candidate sentences using sentiment analysis technique. The experiment determined the threshold of positive and negative affective means, ranging from 0 to 0.03 . Experimental results on Chinese customer reviews show that the final F-score value increased to $87.54 \%$. In addition, a significant difference was set at the 0.01 level, which demonstrates the effectiveness of the technique. The proposed unsupervised method is suitable for the changeable and the large quantity of network review mining. This study does not only meet the need to generalize across different products and various data sizes but also improves the performance in terms of identifying comparative sentences.
\end{abstract}

Keywords: Comparative sentence, Customer reviews, Sentiment analysis, Pattern matching

\section{Introduction}

With the rapid development of e-commerce, online reviews are being recognized as word-of-mouth. According to the social commerce statistics of Kelton Research/Bazaarvoice, $83 \%$ of customers believed that reading user-generated content before making a decision is important in banking and other financial services [1]. Comparative sentences are commonly used in online product reviews to compare product evaluation with other products and thereby convey information that customers are concerned about. By comparing online product reviews, customers may find which product is better; thus, the comparative information has important reference value for decision-making of some potential customers and investors [2]. Product comparisons are not only useful for customers but also enable product manufacturers to improve their products and services.

In recent years, some studies have focused on automatically identifying sentences with comparative meanings by using linguistics, natural language processing, data mining, and other technologies. Relevant studies mainly use two different methods: machine learning and pattern matching method [3]. However, its performance still requires further improvement, and previous research has not performed sentiment analysis in the identification of comparative sentences.

Product reviews reveal customers" "Satisfaction" or "Dissatisfaction," and most of the comparative results are

- E-mail address: lishi@nefu.edu.cn

ISSN: 1791-2377 @ 2016 Eastern Macedonia and Thrace Institute of Technology. All rights reserved. stated in adjectives, which accurately reflect the emotions of customers. Thus, this study performs sentiment analysis to establish a scoring system of sentiment orientation, which will further identify comparative sentences.

\section{State of the Art}

\subsection{Comparative sentences identification}

As a special kind of sentence, comparative sentences have attracted the attention of some linguists. Shu Xiang pointed out that the comparative relationship between different objects must have similar and dissimilar parts, which highlighted the core of comparison [4]. Some researchers currently focus on the automatic identification of comparative sentences and the extraction of comparative relations. The Fourth Chinese Opinion Analysis Evaluation Conference (COAE2012) revealed that comparative sentence identification and factor extraction include two subtasks. In Sub-task 1, comparative sentences are identified from a given sentence set. In Sub-task 2 (optional), the corresponding comparative entities and the corresponding sentiment orientation are extracted from the comparative sentences.

Most studies on the identification of comparative sentences in the English language have combined the pattern matching and machine learning techniques [5],[6],[7]. N Jindal et al. used pattern discovery and supervised machine learning to identify English comparative sentences in which SVM and CSR were used to identify comparative sentences [5], and LSR was used to extract the comparative elements 
[7]. Comparative sentence identification results had $79 \%$ and $81 \%$ precision and recall rate, respectively. Some studies also used machine learning method to carry out experiments [8],[9]. Saritha et al. [10] summarized the techniques used to identify the comparative sentences from text documents. From pattern matching to machine learning, the results of Jindal et al. revealed that most of the techniques still need numerous manual annotations, and their performance requires further improvement.

Several differences exist between English and Chinese in terms of grammar and sentence structure. Chinese expressions are flexible; thus, research on English comparative sentences cannot be duplicated in the Chinese context. Studying Chinese comparative sentences is thus necessary. Many Chinese linguists studied modern Chinese comparative sentences [4],[11] and presented the characteristics of comparative sentences. They discussed comparative linguistic features and statistical characteristics that serve as the basis of linguistics in identifying comparative sentences. Two kinds of research directions in the identification of Chinese comparative sentences exist. One method involves comparing words and patterns, and another adopts machine learning technology to identify sentences.

Based on the comparison of words and patterns, Rui et al [12] developed a Chinese comparison pattern database for identifying comparative sentences. The identification of Chinese comparative sentences was carried out by the comparison of pattern, and the results reached $78.47 \%$ and $79.2 \%$ of the F-score values, respectively. Subsequent studies proposed a rule-based comparative sentence identification technology based on comparison pattern [13], [14], in which the performance was significantly improved. Pattern matching is an unsupervised learning method, which depends on the database used [15]. Other researchers used supervised machine learning technology in identifying comparative sentences [11],[16]. Comparing pattern matching and machine learning, Wei et al. [15] used the SVM model to classify comparative sentences based on the key words, terms, sequence patterns and other features; the model had up to $87.26 \%$ of the F-score value.

The performance of the current research in identifying Chinese comparative sentences requires further improvement. The identification of comparative sentences will significantly affect the review mining process. The product review reflects customers' evaluation, which reflects many consumers' emotions. Therefore, this study considers sentimental factors in comparative sentence identification, which implies that we combine the semantic factor and the comparative model to further improve the performance of identifying comparative sentences based on previous research.

\subsection{Sentiment analysis}

Sentiment analysis technology currently involves machine learning and sentiment analysis [17].

Thus far, most studies used a sentiment dictionary to perform semantics-based sentiment analysis. The sentiment orientation of single sentences and paragraphs were calculated according to the sentiment orientations of the words and was extended to the sentiment orientation of entire articles. The development of an effective sentiment dictionary is the key to the application of the lexicon method in sentiment analysis. The sentiment analysis based on machine learning transforms the sentiment analysis of text into the problem of text classification. It describes the text by expressing the subjective feature of the text, and uses the classical classification method to evaluate the text's sentiment orientation. The key to evaluating the sentiment orientation is to accurately provide the training set and to characterize the text that contains the sentiment orientation.

Several researchers in the English field used the sentiment dictionary to achieve unsupervised or unrequired manual annotation of the training data [18],[19],[20],[21]. In addition, Turney et al. [22] proposed a technology combining Pointwise Mutual Information and Information Retrieval to obtain semantic tendency information through the background database of the search engine. The technology based on machine learning is widely used nowadays [26],[27],[28],[29]. Furthermore, Pang [26] was the first to apply supervised machine learning to sentiment analysis. He compared the results of SVM, Naive Bayesian, and Maximum Entropy in sentiment analysis, and then classified the film reviews in which the precision reached $87.5 \%$.

Most researchers in the Chinese field used the General Inquirer dictionary and HowNet to study sentiment orientation [23],[24]. Based on machine learning, we usually used SVM, Naïve Bayesian, and N-Gram three machine learning algorithms to analyze the sentiment orientation [29] Moreover, some researchers integrated sentiment dictionary and machine learning [25],[30]. Linhong et al. [30] calculated the value of similarity between the possible synonyms and the unknown vocabulary using the semantic similarity formula, from which they obtained the value of sentiment orientation of the vocabulary. Finally they used the SVM to analyze the sentiment orientation of the text.

The researches on the identification of comparative sentence have achieved certain developments, but the performance still needs to be further improved. Although machine learning technology can improve the accuracy, the training examples for each application area need to be manually annotated, which implies plenty of manual work. Some of the most efficient machine learning algorithms, such as SVM and CRF, are unable to produce easily comprehensible results [32]. Moreover, when the classifier is used in other fields, the accuracy of the classification significantly decreases. [33]. For flexible and changeable online reviews, this exploration considers the use of semisupervised or unsupervised technology to identify comparative sentences. Comparative sentences in customer reviews often contain many sentiment factors, and the results of existing studies on sentiment analysis have achieved high accuracy. Thus, this study used technology based on sentiment dictionary to identify comparative sentences from candidate sentences. We used HowNet (CNKI) and Chinese Sentiment Dictionary of Taiwan University as the basic dictionary, and manually added a professional dictionary belonging to the field of Chinese product reviews. We searched the sentiment words, the degree of words, and the negative words in the clauses. The sentiment scores of the candidate sentences were calculated by these words. Finally, we set a threshold to further filter non-comparative sentences with weak sentiment orientation. Through this method, the performance in identifying Chinese comparative sentences was improved.

The rest of this study is organized as follows. Section 3 describes the method for constructing matching pattern database and sentiment analysis, and experiments are carried out to determine the threshold of sentiment orientation. Experimental results and the significance test are presented 
in Section 4. Finally, Section 5 concludes the study and discusses directions for future research.

\section{Methodology}

\subsection{Construction of comparative sentence pattern} database

In this study, we referred to relevant research. Five models, namely, A_C, T_C, S_C, N_C, and H_C were developed from the comparative sentences of manual annotation. We extended and further extracted according to the style of Chinese product reviews. After screening and sorting out, the comparative pattern matching database was constructed and stored in the form of an XML document.

The specific pattern is as follows:

\section{(1) A_C PATTERN}

Examples of patterns:

*( he /cc $\mid$ yu /p $\mid$ tong /p | yu /p ).* (duibi /vi | xiangbi /vi $\mid$ jiao /d $\mid$ jiaozhi /p $\mid$ ru /v $\mid$ xiangdui /b ).*

This pattern deals with common comparative sentences. In this pattern, we classified comparative sentences into equal ratio and different ratio. Generally, the comparison subject, comparison object, and comparison result of this model are evident. The comparison subject is sometimes omitted, but the comparison subject is evident and easy to distinguish. Pronouns can be generally used as replacements.

\section{(2) T_C PATTERN}

Examples of patterns:

*( benlai /d $\mid$ cengjing /d $\mid$ zhiqian /f $\mid$ yiqian /f $\mid / m$ nian /qt qian /f $\mid$ yiwang /t $\mid$ qianduan /t shijian /n | yuanlai /d ).* (xianzai /t $\mid$ muqian /t $\mid$ zhe /rzv c i/qv | benci/r | zhehui ).*

.*( yong /v guo /uguo zhihou /f | yong /v wan /vi yihou /f $\mid$ bi /p yiqian /f $\mid$ bi /p zhiqian /f).*(/a).*

This pattern focuses on the different times and the same product comparisons, focusing on the customers' experience. In previous studies, the comparative sentences of this pattern type were easily overlooked, and the feature words with no clear or relatively stable concentration tended to be neglected. The comparison result of time displacement in non-product review mining is difficult to highlight. In this exploration, the establishment of T_C pattern can be used in product mining, which can obtain valuable information.

\section{(3) S_C PATTERN}

Examples of patterns:

**( zuowei /v | weiju /v | kancheng /v).*( yiliu /b | jipin /n | zhi /uzhi shou /ng | zhi /uzhi wang /n).*

.*(yong /v guo /uguo | yudao /v de /ude1 ).*(zui d).

This pattern corresponds to the "very" category in the classification system of Jianzhong. It refers to the main attributes of the comparison subject in a certain range, which is either best or worst. Comparison of standards of this kind of pattern is often more implied and also brings substantial noise.

\section{(4) N_C PATTERN}

Examples of patterns:

*(bu /d ci /qv yu/p | bu /d yayu /v | bu /d ru /v ).*

This pattern corresponds to the "different radio" category in the classification system of Jianzhong. A difference is observed between the comparative subject and the comparative object. The A_C pattern is different in that the comparison object in the A_C mode precedes the comparison word. The comparison subject and the comparison object in the N_C pattern lie between the comparison words.

\section{(5) H_C PATTERN}

Examples of patterns:

**( meiyou /d | you /vyou ).*(youxiao /a | xiaoguo /n mingxian /ad | youyong /v)

In Chinese, "with" or "no" constitutes a comparative sentence. These comparative sentences also play an important role in the excavation of Chinese product reviews.

We expanded the pattern database using the five models presented. According to the product reviews text crawled, 40 matching patterns are available for manual annotation. Given that only a class of cosmetics products is marked and the Chinese expression is flexible and changing, the current size of our matching database need to be further expanded. It can be extended to other fields, which future work can extend.

In most cases, an inverse relationship is observed between precision and recall, often by adding one, at the expense of the other. To determine whether performance is increasing or decreasing, the two metrics should be combined into a single metric. The F-score value can be used for measurement [34]. Thus, we have Formulas (1), (2), and (3):

$$
\begin{aligned}
& \text { Precision }=\frac{\text { number of extracted comparative sentences }}{\text { number of extracted sentences }} \\
& \text { Recall }=\frac{\text { number of extracted comparative sentences }}{\text { number of comparatives sentences }}
\end{aligned}
$$

F-score $=\frac{2 \times \text { precision } \times \text { recall }}{\text { precision }+ \text { recall }}$

\subsection{Sentiment analysis of comparative sentences}

Through sentiment analysis, the comparative sentences identified by the pattern matching database are subjected to secondary filtering. The process of Sent_Score [4] based on the sentiment dictionary scoring system is shown in Figure 1.

Step 1: We used HowNet (CNKI) and Chinese Sentiment Dictionary of Taiwan University as the basic dictionary, and then manually added a professional dictionary belonging to the field of Chinese product reviews (Table 1). Therefore, positive sentiment dictionary and negative sentiment dictionary were developed. The sentiment words of each candidate comparative sentence were identified using these dictionaries, and the positive or negative position was recorded.

Step 2: When a sentiment word was identified, the degree word was searched from the sentiment word forward. The search ceased when the degree word was identified. A different weight was set for each degree word. The weight of a degree word was multiplied with the sentiment value. 


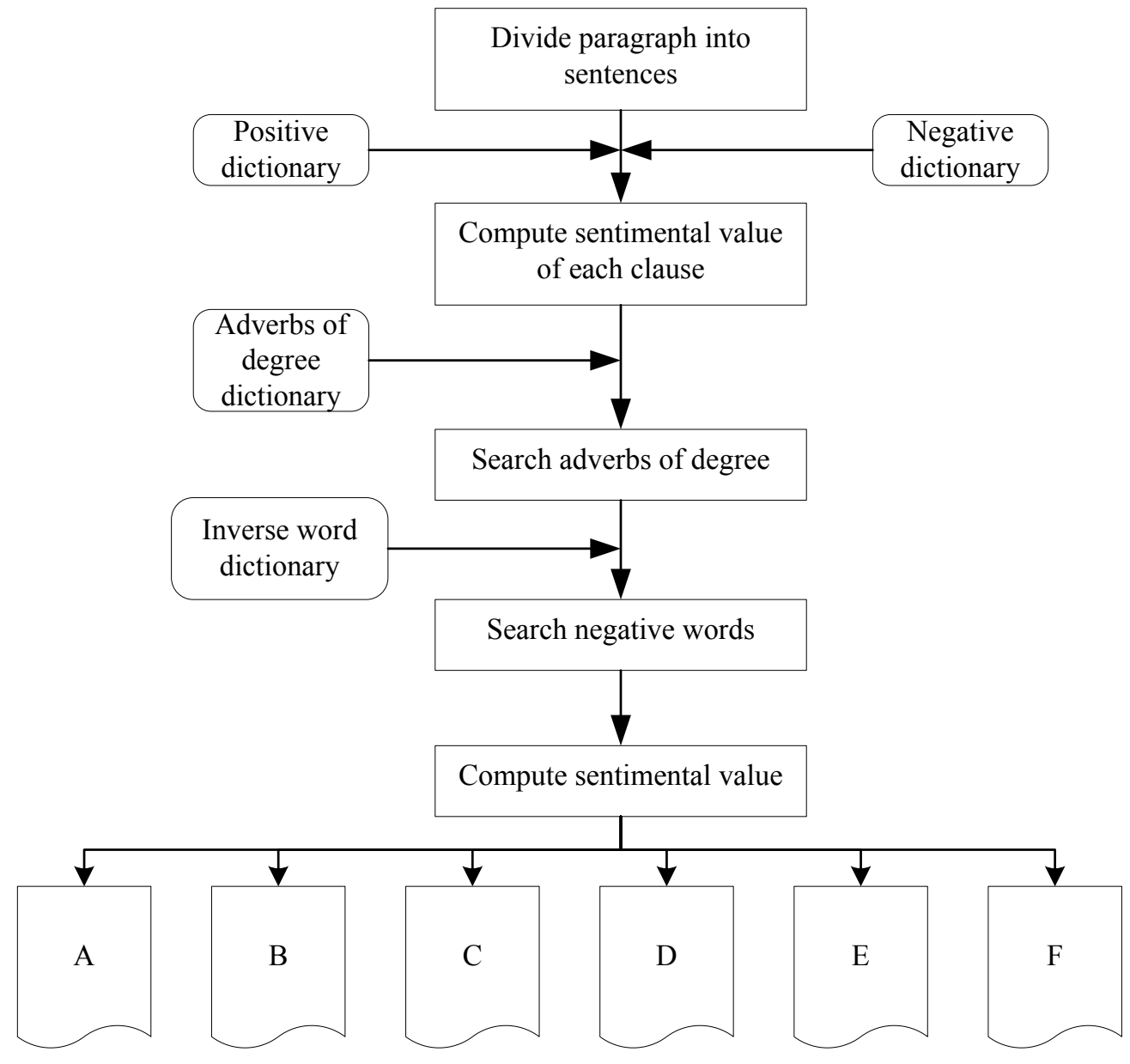

Fig. 1. Flow chart of scoring based on sentiment analysis

Step 3: The negative words were searched from the sentiment word forward. When the number of all negative words was odd, it was multiplied by -1 . When it was even, it was multiplied by 1 .

Step 4: The sentiment value of all the clauses in a review was calculated. We assigned the positive sentiment value to $\mathrm{A}$, and assigned the negative sentiment value to $\mathrm{B}$. Let $\mathrm{C}$ be the positive sentiment mean, and let $\mathrm{D}$ be the negative sentiment mean. The positive affective variance $\mathrm{E}$ and negative affective variance $F$ of each review can be calculated by the clauses.

Step 5: The candidate comparison sentences were secondarily filtered, that is, the candidate comparison sentences were further filtered by setting the threshold value.

Table 1. Professional dictionary added manually

\begin{tabular}{c|c|c|c|c|c|c}
\hline \multirow{2}{*}{ Posdic } & Zhengpin & Zhenpin & Zhenghuo & Baoshi & Xihuan \\
Gaodang & Yiliu & Baobei & Manzu & Ciji & Cijian \\
\hline \multirow{2}{*}{ Negdic } & Posun & Liezhi & Jiahuo & Youni & Citong \\
& Zaogao & Qifen & Chaping & Shanghai & Qipian \\
\hline
\end{tabular}

\subsection{T-test to assess the significance}

We conducted T-test to demonstrate the effectiveness of this technology. If the two results are close to each other, then the results of this study are significantly higher, or the gap is not obvious; thus, we can further verify the effectiveness of the technology [2].

We used two ratios to calculate the T-value using Formula (4) .

$$
\mathrm{T}=\frac{\mathrm{p}-\pi}{\sigma_{\mathrm{p}}}
$$

Among them:

$$
\begin{aligned}
& \sigma_{\mathrm{p}}=\sqrt{\frac{\pi(1-\pi)}{n}} \\
& p=\frac{n_{k}}{n}
\end{aligned}
$$


Significance of $\mathrm{p}$ and $\pi-\left\{\begin{array}{l}\text { Non-significant, }|t|<t_{0.05}(n-2) \\ \text { Significant, } t_{0.05}(n)<|t|<t_{0.01}(n-2) \\ \text { Extremely significant, }|t|>t_{0.01}(n-2)\end{array}\right.$

\subsection{Experiment}

\subsubsection{Data Sources}

The experiments were conducted using cosmetic product reviews because such reviews tend to have many relative components.

We searched and downloaded 1,210 text reviews from the Chinese Amazon.com about Pechoin, Olay, L'Oreal as the three brands in the product reviews. Four human coders were recruited to independently extract comparative sentences from these online reviews. We developed a set of corpus that can cover the comparative sentences in all of these reviews.

\subsubsection{Corpus pretreatment}

At first, we separated each sentence for all the test data. To ensure that follow-up research was based on sentences, this study used the period, exclamation point, question mark, and any combination of the three as a separator for commentary sentence processing. The number of corpus changed for this step is shown in Table 2.

Table 2. Data source and the quantity of experiment data

\begin{tabular}{c|c|c}
\hline Source of Review Corpus & Quantity/Item & Quantity of Clause \\
\hline Essential Water of Pechoin & 400 & 701 \\
Olay's Moisturizer & 400 & 913 \\
L'Oreal Essence Condensation & 410 & 1131 \\
\hline
\end{tabular}

In the second step, we used the Chinese word segmentation tool ICTCLAS (Chinese lexical analysis system of institute of computing technology) to perform word segmentation and POS tagging The experimental data to be filtered were then obtained. Each sentence was stored separately, with its file name as the main code, as the only identification of the follow-up operation. The exploration also excluded the non-punctuation separator reviews text and blank short text. The non-standard review format was provided for the pruning process. For example, no rules were established on continuous space as a statement of the separation; comments only had modal particles, particles, and so on. These sentences account for a small proportion, and its research is meaningless after removal does not affect the follow-up experimental results.

Table 3 shows examples of POS tag types of the ICTCLAS Chinese word segmentation tool.

Table 3. Examples of POS-Mark mapping

\begin{tabular}{c|c|c|c}
\hline Mark & POS & Mark & POS \\
\hline /n & Noun & $/ \mathrm{v}$ & Verb \\
$/ \mathrm{a}$ & Adjective & $/ \mathrm{t}$ & Time-word \\
$/ \mathrm{cc}$ & Conjunction & $/ \mathrm{m}$ & Numeral \\
$/ \mathrm{y}$ & Interjection & $/ \mathrm{p}$ & Preposition \\
\hline
\end{tabular}

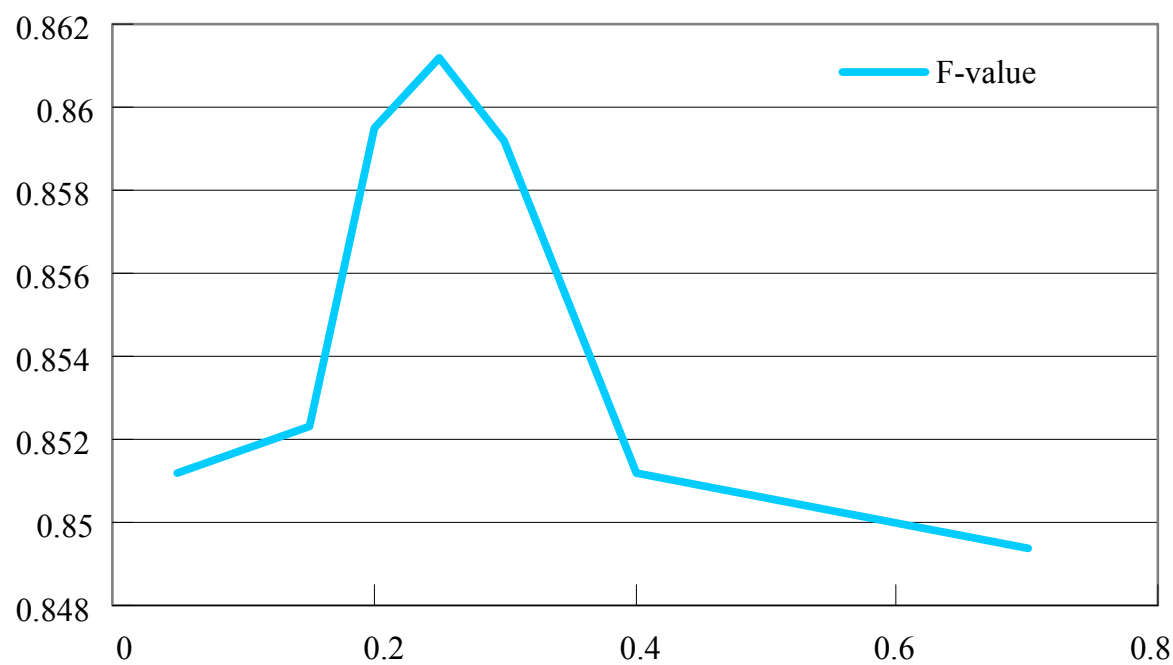

Fig. 2. Effect of threshold settings on F-score values

\subsubsection{Filtering of Comparison Sentences}

We read the content of each review to be filtered as Content [i] in sequence and invoked the filtering test function for 
Content [i] to match the regular expression. If Content [i] matched a pattern in the matching pattern, Pattern [j] matched successfully, returned the true value, and output the comment text number and the serial number of the matching pattern.

In sentiment analysis system Sent_Score, we found the effect of threshold settings on the F-score value of roughly the trend as shown in Figure 2.
The threshold of the absolute value of $\mathrm{C}$ and $\mathrm{D}$ difference was set to [0,0.03], and the threshold of A and B values was set to $[0.25,+\infty]$ in the experiment. Sent_Score system was used to evaluate the scores of the comparative sentences and select according to the requirements.

\subsubsection{Experimental flow chart}

The experiment flow of this study is shown in Figure 3.

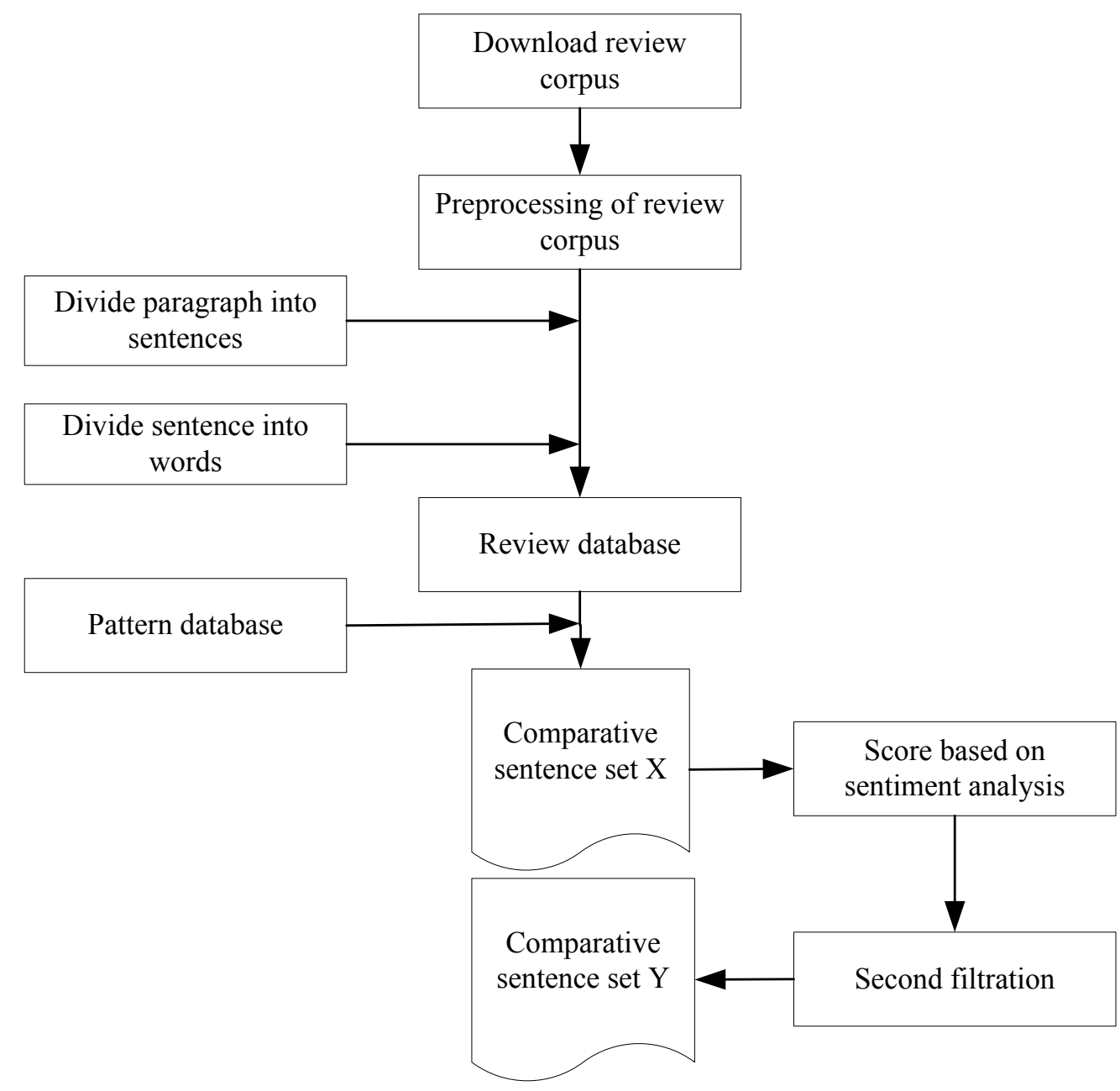

Fig. 3. Proposed method for mining comparative sentences

\section{Result analysis and discussion}

4.1 Filtering results of comparative sentence pattern set Table 4 shows the filtering results of the comparison pattern database based on five basic comparison patterns.

Tab. 4. Results of the dataset's first filtration via patterns of comparative sentence

\begin{tabular}{c|c|c|c}
\hline Product Name & Recall & Precision & F-score \\
\hline Pechoin & 0.9930 & 0.7717 & 0.8685 \\
Olay & 0.9806 & 0.7005 & 0.8172 \\
L'Oreal & 0.9809 & 0.7509 & 0.8506 \\
\hline
\end{tabular}

4.2 Results of secondary filtering of sentiment analysis The Sent Score system is used to evaluate the sentimental degree of the candidate set. The results of the secondary filtering according to the sentiment score are shown in Table 5.
Table 5. Results of the identification of comparative sentences based on sentiment analysis

\begin{tabular}{l|l|l|l}
\hline Product Name & Recall & Precision & F-score \\
\hline Pechoin & 0.9580 & 0.8059 & 0.8754 \\
Olay & 0.9419 & 0.7337 & 0.8249 \\
L'Oreal & 0.9474 & 0.7795 & 0.8553 \\
\hline
\end{tabular}

Our experiment results confirm that the precision has improved significantly after performing sentiment analysis based on the filtering results in the first step. Although the recall rate will be decreased, the F-score value is generally increased. This method improves the performance in identifying comparative sentences.

4.3 Results of this study and other experimental result soft he comparison

For the Chinese product reviews, the technology proposed in this study is compared with the results of other two studies. The analysis is based on the F-score value to comprehensively evaluate the results. 
Table 6. Comparison between this study's experimental results and those of two other studies

\begin{tabular}{c|c|c|c|c}
\hline Indicators & $\begin{array}{l}\text { Experimental results of } \\
\text { this study after first } \\
\text { filtration }\end{array}$ & $\begin{array}{c}\text { Experimental results of } \\
\text { this study after second } \\
\text { filtration }\end{array}$ & $\begin{array}{l}\text { Rui [13] Experimental results based } \\
\text { on comparison pattern }\end{array}$ & $\begin{array}{l}\text { Gao } \\
\text { Experimental results } \\
\text { based } \\
\text { algorithm }\end{array}$ \\
\hline CRM
\end{tabular}

Table 6 shows that the F-score value of this study is higher than that of Rui [13], which is similar to the result of Gao Hui [17] based on machine learning. To a certain extent, machine learning generally performs well [16]. However, supervised machine learning requires review data with proper annotation to build classification models, which is not suitable for the diversification, individuation, and huge amount of network reviews, which will be affected or will require considerable effort. In this study, the use of pattern matching can be better applied to the text of this type of network review.

Empirical evaluation using T-test showed its effectiveness as compared with the results in the study of Rui [13]. In this experiment, the $n$ of precision is the number of all comparative sentences identified, and the $n$ of recall is the number of comparative sentences manual annotation. The average degree of freedom is $n-2$, and $n$ is the sum of the three product reviews. T-test results are shown in Tables 7 and 8 .

Table 7. Results of significance test on precision

\begin{tabular}{c|c|c|c|c|c|c}
\hline Number & $\boldsymbol{p}$ & $\boldsymbol{\pi}$ & $\boldsymbol{n}$ & $\boldsymbol{t}$ value & $\boldsymbol{t}$ value $(\alpha=0.05)$ & $\boldsymbol{t}$ value $(\alpha=0.01)$ \\
\hline $\mathbf{1}$ & 0.8059 & 0.7700 & 170 & 1.1123 & 1.9740 & 2.6050 \\
$\mathbf{2}$ & 0.7337 & 0.7700 & 199 & -1.2168 & 1.9720 & 2.6010 \\
$\mathbf{3}$ & 0.7795 & 0.7700 & 254 & 0.3598 & 1.9690 & 2.5960 \\
\hline Average & 0.7730 & 0.7700 & 623 & -1.6827 & 1.9650 & 2.5840 \\
\hline
\end{tabular}

Table 8. Results of significance test on recall

\begin{tabular}{c|c|c|c|c|c}
\hline Number & $\boldsymbol{p}$ & $\boldsymbol{\pi}$ & $\boldsymbol{n}$ & $\boldsymbol{t}$ value & $\boldsymbol{t}$ value $(\alpha=0.05)$ \\
\hline $\mathbf{1}$ & 0.9580 & 0.8000 & 143 & $4.7235^{* *}$ & 1.9770 \\
$\mathbf{2}$ & 0.9419 & 0.8000 & 155 & $4.4166^{* *}$ & 1.9760 \\
$\mathbf{3}$ & 0.9474 & 0.8000 & 209 & $5.3273^{* *}$ & 2.9720 \\
\hline \multicolumn{2}{|c|}{0.9491} & 0.8000 & 507 & $9.5166^{* *}$ & 1.9650 \\
\hline
\end{tabular}

The overall data experimental results reveal that this approach and Rui's experimental results on precision were not significantly different. However, the recall value yielded a noticeable performance gain. The data indicate that our recall value was better than that of Rui S. The results show that the approach proposed in this study has better comprehensive performance and effectivity.

\section{Conclusions}

To further improve the performance of Chinese comparative sentence identification, this study considered sentiment factors in texts. We proposed a technology of integrating unsupervised sentiment analysis with comparative sentence pattern filtering, and verified the validity of the approach. Finally, the following conclusions can be drawn:

(1)The recall of this approach is significantly higher than the other experimental results, that is, the establishment of this pattern set is correct and efficient.
(2)This study considers that sentiment factors improve the performance of mining results. Although in the experiment the recall decreased. However, the F-score value increased to a certain degree. T-test between the results of this approach and other approaches show that the results are significant at the 0.01 level, indicating that our approach for Chinese Comparative Sentence Identification is effective.

This study performed the unsupervised sentiment analysis to further identify the candidate sets of comparative sentences, which not only improved the mining performance but was also suitable for flexible and changeable network reviews. Extracting such comparative information can help customers make sensible purchasing decisions and help businesses in their product benchmarking efforts. The proposed method has practical significance. Our future work will extend the set of Chinese comparative sentence patterns and focus on how the comparative relation and the comparative elements can be extracted. 


\section{Acknowledgements}

The study was supported by the National Undergraduate

Training Programs for Innovation and Entrepreneurship (No.
201610225054) and the Fundamental Research Funds for the Central Universities (No. 2572015CB33).

\section{References}

1. Shi L, Zhou L, Yijun L., "Improving aspect extraction by augmenting a frequency-based method with web-based similarity measures". Information Processing \& Management, 51(1), 2015, pp.58-67.

2. Huiyun W, Long Y, Shengwei T, Wushouer J, Guanjun F., "Identification of Uyghur comparative sentences". Journal of Chinese Information Processing, 30(1), 2016, pp.148-155.

3. Wang W, Zhao T J, Xin G D, et al., "Exploiting Machine Learning for Comparative Sentences Extraction". International Journal of Hybrid Information Technology, 8(3), 2015, pp.347-354.

4. Ping S., "A Summary of Comparative Sentence System". Applied Linguistics, (s2), 2006, pp.77-80.

5. N Jindal, B Liu., "Identifying comparative sentences in text documents". Proceedings of the 29th Annual International ACM SIGIR Conference on Research and Development in Information Retrieval, New York, USA: ACM, 2006, pp.244-251

6. N Jindal, B Liu., "Mining comparative sentences and relations". Proceedings of the 21st National Conference on Artificial Intelligence, Boston, USA: AAAI, 2006, pp.1331-1336

7. Viveka T., "A Novel Approach Based On Pattern Discovery and Supervised Learning to Identify Comparative Sentences". Automation and Autonomous System, 3(5), 2011, pp.238-246.

8. Liu B, Hu M, Cheng J., "Opinion observer: analyzing and comparing opinions on the web". Proceedings of the 14th international conference on World Wide Web. Chiba, Japan: ACM, 2005, pp.342351.

9. Park D H, Blake C., "Identifying comparative claim sentences in fulltext scientific articles". Proceedings of the ACL 2012 Workshop on Detecting Structure in Scholarly Discourse. Jeju, Korea, 2012, pp.1-9.

10. Saritha S K, Pateriya R K., "Methods for Identifying Comparative Sentences". International Journal of Computer Applications, 108(19), 2014, pp.23-26

11. Huang X, Wan X, Yang J, et al. "Learning to Identify Chinese Comparative Sentences". Journal of Chinese Information Processing, 22(5), 2008, pp.30-38.

12. Song R, Lin H, Chang F. "Chinese comparative sentences identification and comparative relations extraction". Journal of Chinese Information Processing, 23(2), 2009, pp.102-107.

13. Zhou H, Hou M, Hou M, et al., "Chinese Comparative Sentences Identification and Comparative Elements Extraction Based on Semantic Classification". Journal of Chinese Information Processing, 28(3), 2014, pp.136-141.

14. Bai L, Renfen H U, Liu Z., "Recognition of Comparative Sentences Based on Syntactic and Semantic Rules-System". Beijing Daxue Xuebao Ziran Kexue Ban/acta Scientiarum Naturalium Universitatis Pekinensis, 51(2), 2015, pp.275-281.

15. Wang W, Zhao T, Bing X U, et al., "Automatic Identify Chinese Comparative Sentences”. Intelligent Computer \& Applications, 5(5), 2015, pp.1-3

16. Huang $\mathrm{G} \mathrm{H}$, Yao $\mathrm{T}$ F, Liu Q., "Mining Chinese comparative sentences and relations based on CRF algorithm". Application Research of Computers, 27(6), 2010, pp.2061-2064.

17. Schouten K, Frasincar F., "Survey on Aspect-Level Sentiment Analysis". IEEE Transactions on Knowledge \& Data Engineering, 28(3), 2015, pp.1-1.
18. Siersdorfer S, Chelaru S, Nejdl W, et al., "How useful are your comments?: analyzing and predicting youtube comments and comment ratings". Proceedings of the 19th international conference on World wide web. Raleigh, USA: ACM, 2010, pp. 891-900.

19. Taboada M, Brooke J, Tofiloski M, et al., "Lexicon-based methods for sentiment analysis". Computational linguistics, 37(2), 2011, pp.267-307.

20. Rao Y, Lei J, Wenyin L, et al., "Building emotional dictionary for sentiment analysis of online news". World Wide Web, 17(4), 2014, pp.723-742.

21. Moghaddam S, Ester M., "Opinion digger: an unsupervised opinion miner from unstructured product reviews". Proceedings of the 19th ACM international conference on Information and knowledge management. Toronto, Canada: ACM, 2010, pp.1825-1828.

22. Turney $\mathrm{P}$ D, Littman M L., "Measuring praise and criticism: Inference of semantic orientation from association". ACM Transactions on Information Systems (TOIS), 21(4), 2003, pp.315346.

23. Hui N, Zhe R., "Text Affective Feature Extraction for Reviewing Utility Evaluation". New Technology of Library and Information Service, 31(7), 2015, pp.113-121.

24. Wang S, Yang A, Li D., "Research on sentence sentiment classification based on Chinese sentiment word table". Computer Engineering and Applications, 45(24), 2009, pp.153-155.

25. Haihuan Z, Qingsong Y., "Study on the extraction of Chinese microblog subjective sentences based on lexicon and corpus". Journal of East China Normal University(Natural Science), (4), 2014, pp.62-68.

26. Pang B, Lee L, Vaithyanathan S., "Thumbs up?: sentiment classification using machine learning techniques". Proceedings of the ACL-02 conference on Empirical methods in Natural Language Processing, Philadelphia, USA, 2002, pp.79-86.

27. Pak A, Paroubek P., "Twitter as a Corpus for Sentiment Analysis and Opinion Mining". Proceedings of the Seventh conference on International Language Resources and Evaluation (LREC'10), Valletta, Malta, 10, 2010, pp.1320-1326.

28. Barbosa L, Feng J., "Robust sentiment detection on twitter from biased and noisy data". Proceedings of the 23rd International Conference on Computational Linguistics. Beijing, China: Tsinghua University Press, 2, 2010, pp.36-44.

29. Kang H, Yoo S J, Han D., "Senti-lexicon and improved Naïve Bayes algorithms for sentiment analysis of restaurant reviews". Expert Systems with Applications, 39(5), 2012, pp.6000-6010.

30. Linhong X, Hongfei L, Zhihao Y., "Text Orientation Identification Based on Semantic Comprehension". Journal of Chinese Information Processing, 21(1), 2007, pp.96-101.

31. Zhiming L, Lu L., "Empirical study of sentiment classification for Chinese microblog based on machine learning". Computer Engineering and Application. 48(1), 2012, pp.1-4.

32. Liu B., "Sentiment Analysis and Subjectivity". Handbook of natural language processing, 30(36), 2010, pp.627-666.

33. Khan A Z H, Atique M, Thakare V M., "Combining lexicon-based and learning-based methods for Twitter sentiment analysis". International Journal of Electronics, Communication and Soft Computing Science \& Engineering (IJECSCSE), 2015, 89.

34. Shi L, Ming Y., "Mining Frequent and Infrequent Features from Chinese Customer Reviews". Journal of Theoretical and Applied Information Technology, 48(1), 2013, pp.193-199 